\title{
A Method of Traction Partial Jamming on GPS/INS Integrated Navigation
}

\author{
Chen Jun, Liu Yi, Li Yun-hong, Wang Da-ming, An Xin-yuan, \\ Unit 63880 \\ Luoyang, China \\ liuyi_63880@sina.com
}

\begin{abstract}
To solve the GPS/INS integrated navigation countermeasure problem, a method of traction partial jamming on GPS/INS integrated navigation is proposed. Deception jamming signal produced by jamming equipment can force GPS receiver to output navigation and position data that have gradual departure from the real position. When the bias error can not be corrected by integrated navigation, the traction partial jamming occurs. The definition, signal form and simplified form of jamming method are provided in this paper. The hardware-in-theloop simulation verifies the effectiveness of the method and analyses its jamming effects.
\end{abstract}

Keywords-Integrated navigation system, Cruise missile, Complete trajectory simulation

\section{INTRODUCTION}

Global Positioning System (GPS) and Inertial Navigation System (INS) have been tightly combined by the Data Fusion technology ${ }^{[1]}$, which highly improved the anti-jamming capability of navigation. To seek effective jamming methods for GPS/INS Integrated Navigation has become a challenging issue in the field of navigation countermeasure. For now, researches about GPS/INS integrated navigation mostly focus on its algorithm and performance ${ }^{[2]-[8]}$, but few achievements have been published about its countermeasure methods. Based on exhaustive analyses of GPS/INS integrated navigation, a method of traction partial jamming on GPS/INS integrated navigation is proposed in this paper. The definition, signal form and simplified form of jamming method are also provided. The hardware-in-the-loop simulation verifies the effectiveness of the method and analyses its jamming effects.

\section{ANALYSES ON GPS/INS ANTI-JAMMING PERFORMANCE}

In GPS/INS integrated navigation, the anti-jamming capability of GPS can be improved with the data from INS, which is shown by the following. First, the data from INS can be used to distinguish and eliminate the abnormal GPS measurement data due to countermeasures (deception jamming). Second, inertial navigation can help to recapture the signal when GPS loses signal because of movement, jamming or shelter. Third, with INS, the bandwidth of GPS receiver's tracking loop can be quite narrow and the dynamic and anti-jamming performance can be balanced properly.
To revise the error in loose coupling integrated navigation system, the data from GPS should be distinguished firstly. If GPS measurement data is abnormal, it should be abandoned on the first step of data flow. Otherwise, the data can be used as the input of GPS/INS data fusion algorithm.

Some discussions about the method to distinguish the GPS measurement data are as follows. According to the traditional statistics and probability methods, we use $3 \delta$ method to distinguish and eliminate the abnormal measurement errors. The GPS measurement data $Z_{G P S}$ should be distinguished as abnormal data if it satisfies

$$
Z_{G P S}>3 \delta_{\text {ins-gps }}
$$

where $\delta_{\text {ins-gps }}$ is determined by INS error and GPS error. If the errors of INS and GPS are both linear additive, and the average value of GPS error is 0 , we have

$$
\delta_{i n s-g p s}^{2} \approx \delta_{i n s}^{2}+\delta_{g p s}^{2}
$$

In this equation, $\delta_{g p s}$ is the standard deviation of GPS measurement error, $\delta_{i n s}$ is the standard deviation of INS measurement error which is determined by practical INS equipment. Presently, the micro gyros can reach the precision of $0.1 \sim 1^{\circ} / h$, and the precision of micro accelerometers is $1 \sim 10 \mathrm{mg}$. When the fusion period of GPS/INS is $2 \mathrm{~s}, \delta_{\text {ins }}$ is about $4 \sim 40 \mathrm{~m}$.

\section{TRACTION PARTIAL JAMMING ON GPS/INS INTEGRATED NAVIGATION}

A. Definition and theory of the traction partial jamming Definition: deception jamming signal produced by jamming equipment can force GPS receiver to output navigation and position data to gradually depart from the real position. When the bias error $\Delta Z_{G P S}$ satisfies

$$
\Delta Z_{G P S}>3 \delta_{\text {ins }-g p s}
$$

this jamming signal is "traction partial” jamming signal. $\delta_{\text {ins-gps }}$ is determined by INS error and GPS error. 
The distance measurement is the basis of GPS navigation positioning. By measuring time difference, the measurement distance (pseudo range) between the GPS receiver and the satellite can be determined. Because the clock of the client GPS receiver and the satellite are mostly asynchronous, the measurement distance is not the real distance but the one influenced by the asynchrony of clocks.

The pseudo range $\rho^{\prime}$, satellite coordinate $\left(S V x_{i}, S V y_{i}, S V z_{i}\right)$ and GPS receiver coordinate $(R x, R y, R z)$ satisfy the following equation:

$$
\rho^{\prime}=\sqrt{\left(S V x_{i}-R x\right)^{2}+\left(S V y_{i}-R y\right)^{2}+\left(S V z_{i}-R z\right)^{2}}+c \cdot \Delta t_{r}(4)
$$

while the position result can be calculated by

$$
\begin{aligned}
& \sqrt{\left(S V x_{1}-R x\right)^{2}+\left(S V y_{1}-R y\right)^{2}+\left(S V z_{1}-R z\right)^{2}}+B_{1}+C o r r_{1}=\rho_{1}^{\prime} \\
& \sqrt{\left(S V x_{2}-R x\right)^{2}+\left(S V y_{2}-R y\right)^{2}+\left(S V z_{2}-R z\right)^{2}}+B_{2}+C o r r_{2}=\rho_{2}^{\prime} \\
& \sqrt{\left(S V x_{3}-R x\right)^{2}+\left(S V y_{3}-R y\right)^{2}+\left(S V z_{3}-R z\right)^{2}}+B_{3}+C o r r_{3}=\rho_{3}^{\prime} \\
& \sqrt{\left(S V X_{4}-R x\right)^{2}+\left(S V y_{4}-R y\right)^{2}+\left(S V z_{4}-R z\right)^{2}}+B_{4}+C o r r_{4}=\rho_{4}^{\prime}
\end{aligned}
$$

The satellite coordinate $\left(S V x_{i}, S V y_{i}, S V z_{i}\right)$ can be determined by the data of the navigation message from satellites. $B$ is the equivalent distance difference due to the clock error of the GPS receiver. Corr $_{i}$ is the correction of the $i$ th satellite. $\rho_{i}^{\prime}$ is the measurement distance of the $i$ th satellite. The GPS receiver coordinate $(R x, R y, R z)$ and the time difference $\Delta t_{r}$ can be calculated from the expression 4.

To achieve the "traction partial" jamming effects, the measurement distance $\rho_{i}^{\prime}$ from coherent receiving and special calculation should be disturbed effectively by the jamming signal. With the disturbed measurement distance $\rho_{i}^{\prime}$ calculated by jammed signal, the position result and the real position can satisfy the expression 3 .

\section{B. The signal form of the traction partial jamming}

In this section, the mathematical form of the real GPS satellite signal is assumed to be:

$$
r\left(t_{k}\right)=\sqrt{2 P} d\left(t_{k}\right) c\left(t_{k}-\tau\right) \cos \left(\omega t_{k}+\phi\right)+n\left(t_{k}\right)(6)
$$

where $P$ is the power of the signal, $d\left(t_{k}\right)$ is the modulating data, $c\left(t_{k}-\tau\right)$ is the sequence of the false random code, $\omega, \phi$ are the angular frequency and phase of the input signal, $n\left(t_{k}\right)$ is the Additive White Gaussian Noise.

When reaching the GPS receiver, the signal of traction partial jamming should satisfy the following equations.

$$
\left\{\begin{array}{l}
r\left(t_{k}^{\prime}\right)=\sqrt{2 P} d\left(t_{k}^{\prime}\right) c\left(t_{k}^{\prime}-\tau\right) \cos \left(\omega t_{k}^{\prime}+\phi\right)+n\left(t_{k}^{\prime}\right) \\
t_{k}^{\prime}=t_{k}-\Delta t \\
\omega^{\prime}=\omega-\Delta \omega
\end{array}\right.
$$

In these equations, $\Delta t-\frac{\Delta R \cos \theta}{c}, \Delta \omega=\frac{\Delta \nu}{2 \lambda} \cdot \Delta R$ is the difference of the distance between the deception position and GPS satellite and the distance between the real position and GPS satellite. $\Delta v$ is the projection of the difference of the deception velocity and real velocity at the satellite's radial direction. $\theta$ is the tangential angle between the GPS satellite and ground. To achieve the traction partial jamming effects, $\Delta R, \Delta v$ should be proper so that $\Delta Z_{\text {GPS }}$ can satisfy the expression 3 .

Considering the relative position between the jamming equipment and the target receiver, the mathematical form of jamming signal is

$$
\left\{\begin{array}{l}
r\left(t_{k}^{\prime \prime}\right)=\sqrt{2 P} d\left(t_{k}^{\prime \prime}\right) c\left(t_{k}^{\prime \prime}-\tau\right) \cos \left(\omega^{\prime} t_{k}^{\prime \prime}+\phi\right)+n\left(t_{k}^{\prime \prime}\right) \\
t_{k}^{\prime \prime}=t_{k}^{\prime}-\Delta t \\
\omega^{\prime}=\omega-\Delta \omega
\end{array}\right.
$$

In expression $8, \Delta t=\frac{\Delta R^{\prime}}{c}, \Delta R^{\prime}$ is the distance between the jamming equipment and the target receiver.

\section{Simplified form of the traction partial jamming}

According to the former discussion, the time delay and frequency of the jamming signal, in theory, should be controlled accurately to achieve the proper traction partial jamming effects. Since the GPS receiver can receive signals from a couple of satellites, the traction partial jamming system should provide jamming signal for each satellite in theory. However, the effective multiple jamming signals is nearly an exaction in practical applications.

In practical applications, it is not necessary to fully fulfill the requirement of precise multiple jamming signals to achieve the traction partial jamming effects. The position calculation of GPS receiver is based on the measurement distance of a couple of satellites. If the jamming signal can effectively disturb the signal of the key GPS satellite, it can partially achieve the traction partly jamming effects. This conclusion will be proved by the hardware-in-the-loop simulation analyses in the next part.

\section{SimUlATION EXPERIMENT}

To prove the effectiveness of the traction partial jamming, the parameters of the simulation are set as follows. The incoming cruise missile whose speed is $50 \mathrm{~m} / \mathrm{s}$ is simulated by a GPS signal simulation (simulation 1). Another simulation (simulation 2) simulates the 
jamming GPS signal, which is transverse and at the speed of $20 \mathrm{~m} / \mathrm{s}$ and $50 \mathrm{~m} / \mathrm{s}$. The connection of simulation equipments is shown by Figure 1.

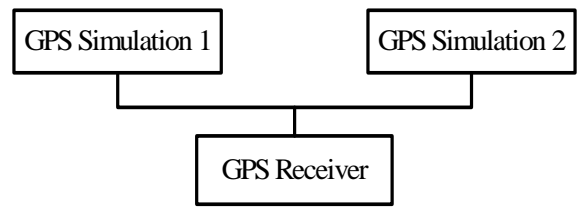

Figure 1. The connection of equipments in traction partial jamming simulation experiment



(a)

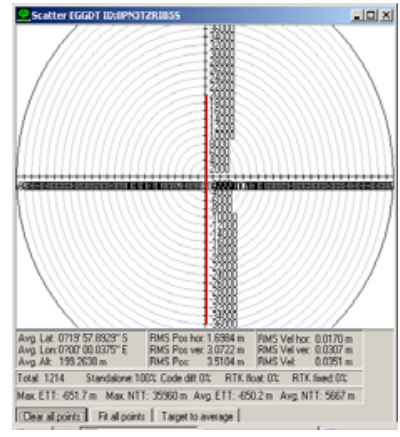

(b)
Figure 2. The preset satellite chart and trajectory in traction partial jamming simulation experiment

The preset trajectory is as follows. The beginning position is $0^{\circ} 10^{\prime}$ north latitude and $0^{\circ} 0^{\prime}$ east latitude. The missile is flying south at speed of $50 \mathrm{~m} / \mathrm{s}$, and the height of the missile stays on $200 \mathrm{~m}$. The beginning time of simulation is set to $12: 00 \mathrm{pm}$, December 2nd, 2012, so the positions of GPS satellites are illustrated as Figure 2(a) and the preset trajectory is illustrated as Figure 2(b).

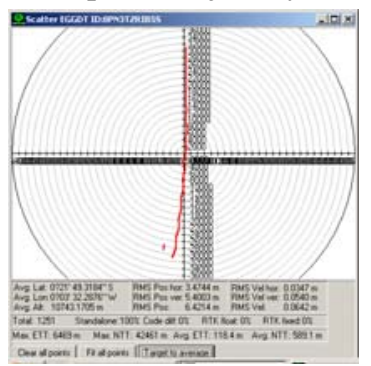

(a)

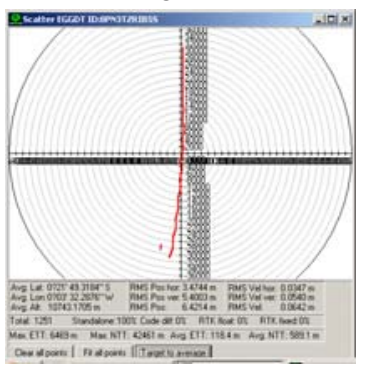

(b)
Figure 3. The 1st and 2nd simulation results of traction partial jamming simulation experiment

When the form of jamming signal is "fly back and forth on a $1 \mathrm{~km}$ runway-like course at speed of $20 \mathrm{~m} / \mathrm{s}$ ” and the deception jamming signal just acts on a single GPS satellite, the simulation trajectory is shown by Figure 3(a). And when the form of jamming signal is "fly back and forth on a $5 \mathrm{~km}$ runway-like course at speed of $50 \mathrm{~m} / \mathrm{s}$ ” and the deception jamming signal just acts on a single GPS satellite, the simulation trajectory is shown by Figure 3(b).

According to the simulation results, the target missile flies normally when it entered the jamming position, the traction partial jamming comes to effect after that. We can also find a negative correlation between the jamming effects and the difference between the deception jamming trajectory and the preset missile trajectory.

\section{ANALYSES ON THE TRACTION PARTIAL JAMMING EFFECTS}

A simulation is designed to analyze the jamming effects of the method proposed further. We assume that in INS, the standard deviation of the gyros drift and the precision of the accelerometers has three numerical levels: $0.1^{\circ} \mathrm{h}, 1 \mathrm{mg} ; 0.5^{\circ} \mathrm{h}, 5 \mathrm{mg} ; 1^{\circ} \mathrm{h}, 10 \mathrm{mg}$. The data of the GPS receiver is refreshed in every $0.1 \mathrm{~s}$ and the position and velocity precision of the receiver are $30 \mathrm{~m}, 0.3 \mathrm{~m} / \mathrm{s}$. The period of the INS data correction by GPS data is 2s. Figure 4(a) shows that the position error of GPS/INS by different INS precision level varies in time. The navigation error cumulates over time and the relationship between the changing rate of navigation error and the INS precision is a positive correlation. If the integrated navigation method is used, the navigation error doesn't cumulate over time, so the navigation precision is highly improved.


(a)

(b)

Figure 4. The analysis about simulation results

When the GPS signal is under the deception jamming, the GPS output data is disturbed. If the rate of change is comparatively slow, the disturbed error cannot be distinguished and eliminated by the expression 1 in the integrated navigation method. The disturbed GPS navigation data keeps correcting the inertial navigation system, so the output data of the integrated navigation method has a gradual departure from the real position eventually.

Now we assume the deception jamming to GPS increases the speed by $1 \mathrm{~m} / \mathrm{s}$ at the north and south direction falsely. The fusion period of GPS/INS is 2s, so the error of the inertial navigation $\delta_{i n s}$ is about $4 \sim 40 \mathrm{~m}$ as the former assuming. The GPS position error due to the deception jamming is about $3 \mathrm{~m}$ which cannot be distinguished and eliminated by the equation 1 . Figure 4(b) 
shows the difference of position error between the GPS/INS and GPS/INS with deception jamming. From the simulation results, we can conclude that the deception jamming can disturb the integrated navigation system effectively and make the missile deviate from the preset trajectory.

\section{CONCLUSIONS}

In this paper, the theory and anti-jamming capability of the GPS/INS integrated navigation is analyzed firstly. The theory analysis, computer simulation and hardware-in-theloop simulation verify the feasibility to jam the integrated navigation system. The method of traction partial jamming is proposed especially. The definition, signal form and simplified form of jamming method are also provided in this paper. The hardware-in-the-loop simulation verifies the effectiveness of the method and analyses its jamming effects.

\section{REFERENCES}

[1] Mohider S.Grewal, Lawrence R.Weill, and Angus P.Andrews. Global Positioning Systems, Inertial Navigation, and Integration, Second Edition [M]. John Wiley\&Sins, Inc, 2007.
[2] CHEN You-rong YUAN Jian-ping An Improved Robust $\mathrm{H}$ Multiple Fading Fault-tolerant Filtering Algorithm for INS/GPS Integrated Navigation [J], in Chinese. Journal of Astronautics, 2009, 30(3):930-936

[3] WANG Yao. More Accurate Methodology for Assessing Multimode GNSS Compatibility [J], in Chinese. Journal of System Engineer and Electronics, 2010, 32(6):1305-1308.

[4] LIU Yi. Passive Radar Guidance Technique and Efficacy Evaluation under Confrontation Condition [D] , in Chinese. National University of Defense Technology, 2010.

[5] J.L.Crassidis. Sigma-point Kalman Filtering for Integrated GPS and Inertial Navigation [J]. IEEE Trans On AES, 2006,42(2):750756

[6] LIN Xue-yuan, JU Jian-bo. GPS/SINS Integrated Navigation Algorithm Based on Neural Network Prediction [J] , in Chinese. Journal of Geometrics and Information Science of Wuhan University, 2011(05).

[7] DOU Chang-jiang. Study of Adaptive Filtering Used by Satellite Navigation Users $[\mathrm{J}]$, in Chinese. Radio Communications Technology, 2006(06).

[8] ZHOU Kun-fang, LI De-wu, ZHOU Xiang-rong. GPS/INS Modular Pattern under Circumstance of Jamming [J]. Journal of Chinese Inertial Technology, 2004(04). 